\title{
Q6ese
}

\section{RESULTADOS DE LA REVISIÓN INTERMEDIA DEL MARCO FINANCIERO PLURIANUAL 2014-2020 Y PERSPECTIVAS PARA EL MARCO FINANCIERO POST-2020}

La Comisión Europea presentó, el 14 de septiembre de 2016, su propuesta de revisión intermedia del marco financiero plurianual (MFP) 2014-2020, la cual fue, posteriormente, enmendada por el Consejo, llegándose a un acuerdo político en principio (unánime con las abstenciones de Italia y Reino Unido) en el Consejo de Asuntos Generales (CAG) del 7 de marzo de 2017. Con posterioridad, el Parlamento Europeo dio su consentimiento al acuerdo en la sesión plenaria del 5 de abril. Estando prevista su adopción formal en el CAG, Cohesión del 25 de abril de 2017, como único trámite pendiente antes de su publicación en el Diario Oficial de la UE para su entrada en vigor, la Presidencia del Consejo retiró el expediente del orden del día del CAG cuando, la víspera del Consejo, Reino Unido manifestó una reserva y, por tanto, se constató la falta de unanimidad necesaria para la adopción formal. En tanto Reino Unido no levante su reserva, el expediente está pendiente de adopción formal.

El objetivo de la revisión, una vez analizado el funcionamiento del marco en los tres primeros años del periodo, es dotar de la máxima flexibilidad posible al presupuesto comunitario para permitir la rápida financiación de situaciones no previstas cuando se adoptó el MFP en 2013, tales como las recientes crisis migratoria y de seguridad en la UE. Tras meses de negociación, el acuerdo final contiene una flexibilidad algo menor que la deseada por la Comisión pero suficiente para lo que resta de este MFP y garantiza a los EE MM cierto grado de predictibilidad a efectos de elaboración de sus respectivos presupuestos nacionales.

De cara a la elaboración del próximo MFP post 2020, se partirá de una UE sin Reino Unido y unas circunstancias económicas y financieras diferentes a las del marco anterior.

Palabras clave: presupuesto UE, instrumentos especiales, instrumento de flexibilidad, Iniciativa de Empleo Juvenil, Fondo Europeo de Inversiones Estratégicas.

Clasificación JEL: H3, H6, H7.

\section{Introducción}

El Parlamento y el Consejo alcanzaron un acuerdo político el 27 de junio de 2013 sobre el

*Este artículo ha sido elaborado por M. ․ Victoria Carcelén Ordóñez. Subdirectora General Adjunta de Asuntos Económicos y Financieros. Secretaría de Estado para la Unión Europea. Ministerio de Asuntos Exteriores y Cooperación.

Versión de abril de 2017. marco financiero plurianual (MFP) para 20142020. Su adopción formal por el Consejo se produjo unos meses más tarde, el 2 de diciembre de 2013. Para su elaboración se tuvieron en cuenta las circunstancias y datos económicos disponibles en fechas previas a su adopción, en un contexto en el que las finanzas públicas estaban sometidas a estrictas políticas de $\triangleright$ 
austeridad en muchos de los Estados miembros (EE MM). Por ello, el MFP fijó, por primera vez, unos techos de gasto inferiores a los del previo MFP 2007-2013 y se centró sobre todo en financiar políticas con un valor añadido de la UE que fomentasen el crecimiento y el empleo.

El Reglamento 1311/2013, por el que se establece el marco financiero plurianual para el período 2014-2020, recoge en su artículo 7, ante la incertidumbre de que los escenarios económicos y sociales utilizados en 2013 pudiesen variar durante el período de vigencia del marco, la obligación de la Comisión de realizar en 2016 una revisión y ajuste de las dotaciones de la política de cohesión teniendo en cuenta los datos estadísticos más actualizados de los que se disponga. El importe neto total del ajuste no podía superar los 4.000 millones de euros (a precios de 2011) y se repartiría entre aquellos EE MM cuya divergencia acumulativa del producto interior bruto (PIB) nacional fuese superior a +/- 5 por 100. España ha sido el país más beneficiado por el ajuste, asignándole 1.800 millones de euros (a precios de 2011) del total, seguido por Italia y Grecia.

Por otro lado, teniendo en cuenta que el Parlamento que dio su consentimiento en 2013 al MFP 2014-2020 iba a ser renovado en las elecciones de mayo de 2014 y el hecho de que pudieran surgir nuevos desafíos y prioridades políticas en los años posteriores, se introdujo en el artículo 2 del Reglamento MFP la necesidad de realizar un examen/evaluación de su evolución a medio plazo (en 2016) al objeto de poder detectar posibles deficiencias del marco en su funcionamiento durante sus tres primeros ejercicios, y si fuese el caso, proponer mejoras.

En cumplimiento de lo dispuesto, la Comisión presentó el 14 de septiembre de 2016 su propuesta de revisión del MFP 2014-2020, que implica modificaciones de varios textos legislativos ${ }^{1}$. Además, la propuesta de revisión intermedia se presentaba acompañada de una propuesta ómnibus de revisión del Reglamento 966/2012 sobre las normas financieras aplicables al presupuesto general de la Unión y varios actos de base ${ }^{2}$.

\section{Estructura del MFP}

A efectos de una mayor comprensión de la propuesta de la Comisión, a continuación se define y detalla brevemente la estructura del MFP.

El MFP, regulado en el artículo 312 del Tratado de Funcionamiento de la UE (TFUE) que establece un periodo mínimo de 5 años (actualmente de 7 años), fija los límites de gasto máximo por políticas que cada año pueden dotarse en el presupuesto anual, tanto para créditos de compromiso como créditos de pago. Los créditos de compromiso son el importe de las obligaciones legales que como máximo puede contraer la UE en un año y que pueden gastarse en ese mismo año o en $\triangleright$

\footnotetext{
Reglamento 1311/2013, por el que se establece el marco financiero plurianual para el período 2014-2020, propuesta de modificación de la Decisión n.ำ 2015/435 del Parlamento Europeo y el Consejo de 17 de diciembre de 2014 sobre la movilización del margen para imprevistos.

Propuesta de modificación del Acuerdo Interinstitucional de 2 de diciembre de 2013, entre el Parlamento Europeo, el Consejo y la Comisión sobre disciplina presupuestaria, cooperación en materia presupuestaria y buena gestión financiera

Propuesta de Reglamento del Parlamento Europeo y del Consejo

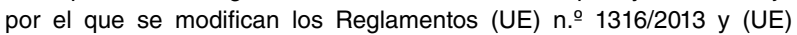
n. ${ }^{\circ}$ 2015/1017 por lo que se refiere a la prórroga de la vigencia del Fondo Europeo de Inversiones Estratégicas, así como la introducción de mejoras técnicas para dicho Fondo y el Centro Europeo de Asesoramiento para la Inversión.

2 Propuesta de Reglamento del Parlamento Europeo y del Consejo sobre las normas financieras aplicables al presupuesto general de la Unión y por el que se modifican los Reglamentos (UE) n.․ 1296/2013, (UE) n. ${ }^{1299 / 2013}$, (UE) n.. 1301/2013, (UE) n. $.1303 / 2013$, (UE) n. $.1304 / 2013$, (UE) n.․ 1305/2013, (UE) n. 1306/2013, (UE) n. 1307/2013, (UE) n. ${ }^{1308 / 2013, ~(U E) ~ n . o ~ 1309 / 2013, ~(U E) ~ n . o ~ 1316 / 2013, ~(U E) ~ n . o ~ 223 / 2014, ~}$

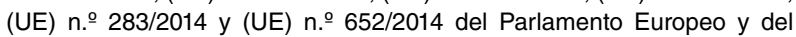
Consejo, y Decisión 541/2014/UE del Parlamento Europeo y del Consejo.

Reglamento del Parlamento Europeo y del Consejo por el que se

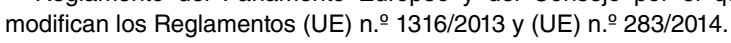


posteriores. Los créditos de pago recogen el gasto efectivo anual.

Su estructura se divide en seis categorías de gasto (rúbricas, headings en inglés) que corresponden a distintas políticas de la UE (ver anexo con los importes correspondientes).

Rúbrica 1. Crecimiento inteligente e inclusivo: 1a. Competitividad para el crecimiento y el empleo: abarca la financiación de investigación, desarrollo e innovación; educación y formación; inversiones en redes transeuropeas de energía, transporte y telecomunicaciones; política social; desarrollo de empresas, etcétera. 1b. Cohesión económica, social y territorial: abarca la política regional, que tiene como fin ayudar a los países y regiones menos desarrollados de la UE a avanzar, aumentar su competitividad y fomentar la cooperación interregional.

Rúbrica 2. Desarrollo sostenible. Recursos naturales: incluye la política agrícola común (PAC), la política pesquera común, desarrollo rural y medidas medioambientales. Es la rúbrica con mayor dotación del presupuesto, junto con la $1 \mathrm{~b}$.

Rúbrica 3. Seguridad y ciudadanía. Engloba la financiación de justicia y asuntos de interior, protección de las fronteras, política de inmigración y asilo, salud pública; protección de los consumidores, juventud, cultura, información y diálogo con los ciudadanos.

Rúbrica 4. Una Europa global. Abarca toda la acción exterior (política exterior) de la UE, como la ayuda al desarrollo y la ayuda humanitaria.

Rúbrica 5.Administración. Cubre los gastos administrativos de todas las instituciones europeas, las pensiones y las escuelas europeas.

Rúbrica 6. Compensaciones. Recoge un mecanismo temporal de tesorería destinado a garantizar que Croacia, miembro de la UE desde julio de 2013, no contribuya más al presupuesto de la UE de lo que obtenga, en virtud del mismo, en el primer año siguiente a su adhesión.

\section{Propuesta de la Comisión de revisión del MFP 2014-2020}

Habiendo examinado el funcionamiento de los primeros ejercicios del MFP, la propuesta de revisión de la Comisión tiene en cuenta el nuevo ámbito económico y social, así como los nuevos desafíos en Europa, consecuencia, principalmente, de los masivos flujos migratorios procedentes de otros continentes y la crisis de seguridad con origen en diversos ataques terroristas.

Por otro lado, partiendo de la base de que los límites de gasto de las rúbricas 3 y 4 del MFP son escasos $\mathrm{y}$, para dar respuesta a la crisis migratoria, en los ejercicios previos se habían agotado, y han requerido el uso de la máxima flexibilidad presupuestaria posible, la Comisión, al objeto de poder reaccionar con celeridad y dotar de financiación suficiente a las nuevas prioridades políticas, propone incrementar la flexibilidad presupuestaria y reforzar las dotaciones de determinadas partidas para el presupuesto de 2017 y los ejercicios 2018-2020.

En la propuesta podemos distinguir dos aspectos: por un lado, la parte legislativa, es decir, las enmiendas que se proponen a la legislación vigente para dar cabida a las demandas de la Comisión; y por otro lado, la propuesta $\triangleright$ 
económica, es decir, el impacto presupuestario que la propuesta tiene, no solo en el presupuesto para 2017, sino también para el resto de periodo y su fuente de financiación. Ambas partes forman un paquete único de negociación.

\subsection{Propuesta económica}

La propuesta económica, por un importe total de 6.334 millones de euros, tiene por objeto reforzar la financiación de nuevas prioridades políticas (migración y seguridad, crecimiento y empleo) e implica el incremento de la dotación presupuestaria en las siguientes partidas del Presupuesto UE para $2017^{3}$, y los ejercicios 2018-2020.

Rúbrica 1a. Competitividad para el crecimiento y el empleo. Presenta un incremento de 1.400 millones de euros que se destinarán a seguir impulsando el crecimiento y el empleo a través de una mayor financiación para programas de alto rendimiento, incluyendo la ampliación/prórroga del Fondo europeo de inversiones estratégicas (FEIE) ${ }^{4}$.

Rúbrica 1b. Cohesión económica, social y territorial. Recoge 1.000 millones de euros adicionales para la Iniciativa de Empleo Juvenil (YEI, siglas en ingles).

Rúbrica 3. Seguridad y ciudadanía. Con un aumento de 2.549 millones de euros, que se destinarán a apoyar las actividades en curso en los ámbitos de la migración, la seguridad y el control de las fronteras

\footnotetext{
3 Hay que tener en cuenta que cuando se presentó la propuesta de revisión del MFP aún no se había acordado por parte del Parlamento y el Consejo el Presupuesto de la UE para 2017. El Consejo ECOFIN, presupuesto del 13 de noviembre de 2016, adoptó su posición sobre el presupuesto 2017 por mayoría cualificada tras el proceso de conciliación con el Parlamento.

4 Expediente con acuerdo político en el Consejo ECOFIN del 6 de diciembre de 2016, en la actualidad en fase negociadora con el Parlamento.
}

exteriores, incluida la creación de la Guardia Europea de Fronteras y Costas, la Agencia de Asilo de la UE y la reforma del Sistema Europeo Común de Asilo.

Rúbrica 4. Europa global. Refuerzo de 1.385 millones de euros, de los cuales 250 millones se destinarán al nuevo Fondo Europeo de Desarrollo Sostenible en el marco del Plan de Inversiones en el Exterior ${ }^{5}$, que apoyará las inversiones en regiones fuera de la UE e incentiva la inversión privada para abordar en el origen la cuestión migratoria.

CUADRO 1

RESUMEN DE LA PROPUESTA ECONÓMICA

\begin{tabular}{|l|c|}
\hline \multicolumn{1}{|c|}{ Rúbricas } & $\begin{array}{c}\text { Propuesta de la Comisión } \\
\text { Créditos de compromiso } \\
\text { (millones de euros) }\end{array}$ \\
\hline Rúbrica 1a & \\
$\quad$ Horizonte 2020 & 400 \\
CEF Transporte & 400 \\
Erasmus+ & 200 \\
COSME & 200 \\
Wifi4EU & 50 \\
FEIE & 150 \\
Total Rúbrica 1a & 1.400 \\
Rúbrica 1b (YEI) & 1.000 \\
Rúbrica 3 & 2.549 \\
Rúbrica 4 & 1.385 \\
\hline Total Rúbricas 1a, 1b, 3, 4 & $\mathbf{6 . 3 3 4}$ \\
\hline Fuente: COM 2016 (603). & \\
\hline
\end{tabular}

La financiación de estos incrementos se realizaría con cargo a una mayor aportación por parte de los EE MM al Presupuesto UE (conforme a los márgenes disponibles).

\subsection{Propuesta legislativa}

La propuesta legislativa implica la modificación de los textos legislativos señalados en $\triangleright$

5 Expediente con acuerdo político en el Consejo de Asuntos Generales de 13 de diciembre 2016, en la actualidad en fase negociadora con el Parlamento. 
la introducción, y en concreto afectan a los siguientes aspectos:

Margen global de pagos. Es la diferencia entre los pagos ejecutados y los techos de créditos de pagos del MFP para cada año (n). El artículo 5 del Reglamento 1311/2013 fija unos los límites máximos de créditos de pago a precios de 2011 de 7.000 millones de euros en 2018, 9.000 millones de euros en 2019 y 10.000 millones de euros en 2010, que deben ser respetados en los presupuestos anuales de la UE. La propuesta de la Comisión implica eliminar los límites para tener más margen disponible de gasto.

Margen global de compromisos. Es la diferencia entre los créditos fijados en el MFP y los créditos aprobados en el presupuesto anual. El artículo 14 del Reglamento 1311/2013 establece que los márgenes de compromisos procedentes de los ejercicios 2014-2017 podrán utilizarse en los ejercicios 2017-2020, pero su uso está restringido para objetivos de políticas relacionadas con el crecimiento y el empleo, y en particular el empleo juvenil. La propuesta de la Comisión implica suprimir las restricciones, tanto la temporal como la de su ámbito de aplicación, y, por tanto, ampliar el ámbito para poder utilizarlo para financiar cualquier política.

Se propone aumentar el importe máximo anual establecido para algunos de los instrumentos especiales: la Reserva de Ayuda de Emergencia se incrementaría desde $280 \mathrm{mi}-$ llones de euros a 500 millones de euros (a precios de 2011) y el importe del Instrumento de Flexibilidad pasaría de los 471 millones de euros (a precios de 2011) de la actualidad a 1.000 millones de euros (a precios de 2011).

Si bien la propuesta de la Comisión solo afecta a estos instrumentos para una visión general de la flexibilidad vigente, a continuación se detalla la lista de instrumentos especiales, su finalidad y su dotación presupuestaria actual.

La Comisión propone reforzar los instrumentos especiales con la creación de un nuevo Instrumento: la Reserva de Crisis de la Unión Europea que viene a añadirse a los instrumentos especiales previstos en la actualidad y que permitirá a la Unión reaccionar rápidamente a las crisis que se presenten. Se nutrirá de créditos descomprometidos en los dos años previos (n-2) y se utilizará hasta el año inmediato posterior $(n+1)$.

Por último, propone también modificar el Acuerdo Interinstitucional de 2 de diciembre de 2013, en consecuencia, para incluir el nuevo instrumento especial, la reserva de crisis de la UE y, por otro lado, y aprovechando la revisión intermedia, modificar la Decisión n.ำ 2015/435 del Parlamento Europeo y el Consejo de 17 de diciembre de 2014 sobre la movilización del margen para imprevistos, para compensar en 2017 el importe de créditos de pago utilizados en 2014 (2.818 millones de euros), en vez de compensarlo en 2018-2020, tal y como se acordó en 2014.

RESUMEN DE LOS INSTRUMENTOS ESPECIALES Y SU CUANTÍA

\begin{tabular}{|l|c|c|}
\hline Instrumentos especiales en $\mathbf{2 0 1 6}$ (artículos de 9 a 12 del Reglamento MFP) & $\begin{array}{c}\text { Precios 2011 } \\
\text { (millones de euros) }\end{array}$ & $\begin{array}{c}\text { Ejercicios en los que } \\
\text { se puede gastar }\end{array}$ \\
\hline Reserva de Ayuda de Emergencia & 280 \\
Fondo de Solidaridad & 500 \\
Instrumento de Flexibilidad & 471 \\
Fondo Europeo de Adaptación a la Globalización & 150 \\
TOTAL & 1.401 \\
\hline Fuente: Reglamento 1311/20103. & \\
\hline
\end{tabular}




\section{Instrumentos especiales}

- La Reserva para Ayuda de Emergencia tiene como objetivo permitir una respuesta rápida a las necesidades concretas de ayuda de terceros países generadas por acontecimientos imprevisibles al establecerse el presupuesto, prioritariamente para acciones de carácter humanitario, pero también, cuando las circunstancias así lo exijan, para la gestión de las crisis civiles y la protección civil, así como para situaciones de especial presión resultantes de los flujos migratorios en las fronteras exteriores de la Unión.

- La finalidad del Fondo de Solidaridad de la Unión Europea es prestar asistencia financiera en caso de que se produzca una catástrofe importante en el territorio de un Estado miembro o de un país candidato.

- El Instrumento de Flexibilidad está destinado a permitir la financiación, en un determinado ejercicio presupuestario, de gastos claramente determinados que no pudieran financiarse dentro de los límites máximos disponibles para una o más de las demás rúbricas.

- El objeto del Fondo Europeo de Adaptación a la Globalización (FEAG) es prestar apoyo adicional a los trabajadores despedidos a raíz de importantes cambios estructurales en los patrones del comercio mundial como consecuencia de la globalización y ayudarlos a reincorporarse al mercado de trabajo.

\section{Desarrollo de las negociaciones}

Desde el inicio de las negociaciones, bajo presidencia eslovaca en el Consejo durante el segundo semestre de 2016, surgieron dos grupos diferentes de países con similares posiciones:

- Por un lado, aquellas delegaciones (integradas mayoritariamente por países cohesión) que abogaban por una mayor flexibilidad, apoyando la propuesta de la Comisión y defendiendo que el cómputo de los créditos de pago de los instrumentos especiales se integre dentro de los techos del MFP.

- Por otro, el grupo de delegaciones (formado, sobre todo, por contribuyentes netos) que sostiene que el MFP cuenta con dotación suficiente y goza de la necesaria flexibilidad, y aboga por el cómputo de los créditos de pagos de los instrumentos especiales dentro de los techos máximos de gasto del MFP.

Esta última cuestión constituyó un elemento importante y central de las negociaciones, ya que, si los créditos de pago se computan fuera de los techos del MFP, se incrementa el gasto efectivo, pero, por otro lado, aportaría más flexibilidad al gasto.

La negociación se prolongó hasta el 13 de diciembre de 2016, cuando en el Consejo de Asuntos Generales la propuesta de compromiso de la presidencia obtuvo el apoyo/conformidad de todas las delegaciones, excepto la abstención de Reino Unido y una reserva de Italia.

Este expediente tiene una tramitación legislativa especial, conforme al artículo 312 del Tratado de Funcionamiento de la UE (TFUE), que requiere unanimidad en el Consejo y que el Parlamento 6 dé su visto bueno al acuerdo.

Llegados a este punto, con ausencia de unanimidad, los esfuerzos de la presidencia se centraron en intentar que Italia levantase cuanto antes su reserva, teniendo siempre presente que cualquier ligero cambio en $\triangleright$

6 A efectos de facilitar el voto favorable del acuerdo final en el Parlamento, durante la negociación del expediente en el Consejo, la Presidencia ha mantenido contactos informales con el Parlamento. 
su propuesta de compromiso podría alterar el equilibrio alcanzado e implicar que otras delegaciones reconsiderasen su posición final.

Estancado el expediente desde diciembre de 2016, había cierta urgencia para su aprobación antes de que Reino Unido activase el artículo 50 del Tratado de Lisboa para su salida efectiva de la UE; las circunstancias hacían pensar que lo que hasta ahora había sido una abstención de Reino Unido podría transformarse, una vez activado el artículo 50, en un voto en contra que rompería la necesaria unanimidad.

Tras varios intentos de la nueva presidencia maltesa del Consejo (entrante en enero de 2017) por conseguir el acuerdo, en la víspera de la reunión del Consejo de Asunto Generales (CAG) del 7 de marzo de 2017, la Comisión circuló una declaración que atendía la petición de la delegación italiana de abrir la posibilidad de que los créditos de la YEI acordados en la revisión pudieran incrementarse si fuese necesario más allá de los 1.200 millones de euros acordados, y siempre que hubiese posibilidades de financiación.

Después de meses de negociaciones, en el CAG del 7 de marzo de 2017, Italia levantó su reserva y se llegó a un acuerdo en principio unánime (con la abstención de Reino Unido e Italia) en los términos que se detallan en el apartado siguiente.

\section{Contenido del acuerdo unánime en el Consejo}

El Acuerdo sobre la revisión intermedia del MFP 2014-2020 garantiza un cierto nivel de flexibilidad presupuestaria adicional que permite reaccionar ante circunstancias imprevistas, pero reduce las pretensiones iniciales de la Comisión ante el rechazo de los EE MM a incrementar el gasto y la necesidad de poder garantizar cierto grado de predictibilidad en la elaboración y dotación de sus presupuestos nacionales. Reduce también los importes propuestos para reforzar determinadas líneas y políticas, y establece que parte de su financiación se realice mediante redistribución de otros créditos del presupuesto, para de esta manera no incrementar las aportaciones de los EE MM al Presupuesto UE. Incluye, además, una serie de declaraciones conjuntas del Consejo y el Parlamento, que precisan determinados aspectos de los elementos del acuerdo y la antes citada declaración de la Comisión sobre la YEI.

\subsection{Parte económica}

Como se ha indicado anteriormente, las cantidades iniciales se han reducido, con un importe acordado de 6.009 millones de euros, frente a los 6.334 millones de euros inicialmente previstos, cuyo detalle se incluye en el Cuadro 3.

\section{CUADRO 3}

COMPARATIVA PROPUESTA ECONÓMICA

(Créditos de compromiso, millones de euros)

\begin{tabular}{|l|c|c|}
\hline \multicolumn{1}{|c|}{ Rúbricas } & $\begin{array}{c}\text { Propuesta de } \\
\text { la Comisión }\end{array}$ & Acuerdo final \\
\hline Rúbrica 1a & & \\
Horizonte 2020 & 400 & 200 \\
CEF Transporte & 400 & 300 \\
Erasmus+ & 200 & 100 \\
COSME & 200 & 100 \\
Wifi4EU* & 50 & 25 \\
FEIE* & 150 & 150 \\
Total Rúbrica 1a & 1.400 & 875 \\
Rúbrica 1b (YEI) & 1.000 & $1.200^{\star *}$ \\
Rúbrica 3 & 2.549 & 2.549 \\
Rúbrica 4* & 1.385 & 1.385 \\
\hline Total Rúbricas 1a, 1b, 3, 4 & $\mathbf{6 . 3 3 4}$ & $\mathbf{6 . 0 0 9}$ \\
\hline * Sin prejuzgar el resultado de las discusiones en proceso \\
sobre los proyectos legislativos de las rúbricas 1a y 4. \\
** Distribuidos a lo largo de 4 años (2017-2020). \\
Fuente: COM 2016 (6030) y ST7030/17 y ST7031/17 ADD2. \\
\hline \multicolumn{2}{|l|}{}
\end{tabular}


El acuerdo implica que se han reducido exclusivamente los incrementos propuestos en las partidas de la rúbrica $1 \mathrm{a}$, por considerarse no suficientemente justificados, y se ha incrementado, atendiendo la petición de varios EE MM (incluidos España e Italia), la dotación de la YEI en 200 millones de euros adicionales, lo que hace un total de 1.200 millones de euros a distribuir en cuatro años.

En una de las declaraciones conjuntas, antes citadas, se precisa, además, que parte de la financiación de estos incrementos, en concreto 945 millones de euros, se financiarán con redistribución de créditos provenientes de la rúbrica 1a, 875 millones de euros y 70 millones de euros de la rúbrica 4, en lugar de con nuevas aportaciones de los EE MM, tal y como proponía la Comisión inicialmente.

\subsection{Parte legislativa}

Por un lado, se ha reducido la flexibilidad propuesta por la Comisión e implica que la propuesta de la nueva reserva de crisis desaparece, pero, por otro, se ha buscado una nueva vía para reutilizar los instrumentos especiales, todo ello en los siguientes términos.

En relación a la propuesta de la Comisión sobre los límites del margen global para pagos, en vez de suprimirse todos los límites, se aumenta el importe del máximo de los ajustes anuales de los ejercicios 2019 y 2020 . Pasan de 9.000 millones de euros y 10.000 millones de euros a 11.000 millones de euros y $13.000 \mathrm{mi}-$ llones de euros, respectivamente; de este modo, a pesar de no aceptar la propuesta inicial de la Comisión, se admite incrementar los limites existentes.

Sobre el margen global para compromisos, el acuerdo final elimina la limitación temporal y, en vez de suprimir las restricciones del ámbito de aplicación, tal y como proponía la Comisión, amplía las restricciones de uso del margen también para migración y seguridad, además del crecimiento del empleo, en concreto la YEI, que ya está vigente.

En lo que respecta a los instrumentos especiales, se incrementan los importes sin alcanzar las cifras propuestas por la Comisión. La Reserva de Ayuda de Emergencia se incrementa de $\mathbf{2 8 0}$ millones de euros a $\mathbf{3 0 0}$ millones (a precios de 2011) y el Instrumento de Flexibilidad pasa de los 471 millones de euros de la actualidad a 600 millones (a precios de 2011).

Como novedad propuesta por el Consejo frente a la propuesta inicial de la Comisión, y en lugar de crear un nuevo instrumento especial (Reserva de Crisis), se establece la reutilización de los instrumentos especiales actuales: ello implica que la dotación anual del Instrumento de Flexibilidad se podrá incrementar por encima de los 600 millones de euros para 2017 en los siguientes términos:

1) Con una cantidad equivalente a la parte del importe anual del Fondo de Solidaridad que haya quedado cancelada en el ejercicio anterior.

2) Con una cantidad equivalente a la parte del importe anual del Fondo Europeo de Adaptación a la Globalización que haya quedado cancelada en el ejercicio anterior.

Ambos importes, con la legislación actual, no se podían utilizar y se perdían.

En cuanto al cómputo de los créditos de pago de los instrumentos especiales dentro/ fuera de los techos del MFP, dada la falta de consenso en el Consejo en este punto, se acuerda dejar la cuestión abierta y se decidirá caso por caso cuando sea necesario.

El resto de elementos de la propuesta relacionados con la compensación del margen $\triangleright$ 
de imprevistos se acepta en los términos propuestos por la Comisión.

La propuesta acordada unánimemente (acuerdo en principio) por el Consejo sobre la revisión intermedia del MFP 2014-2020 fue sometida a votación del Parlamento Europeo para su consentimiento en la sesión plenaria del 5 de abril, basándose en la recomendación favorable emitida por la Comisión de presupuesto (COBU del 27 de abril) y adoptada por mayoría.

Una vez obtenido el consentimiento del Parlamento, conforme al TFUE, el expediente iba a adoptarse formalmente en el CAGcohesión del 25 de abril y, posteriormente, ser publicado en el Diario Oficial de la UE para su entrada en vigor. No obstante, la Presidencia del Consejo retiró el expediente del orden del día la víspera del CAG, cuando constató la ausencia de unanimidad, ante la reserva manifestada por Reino Unido hasta que no se celebren las elecciones en el país el 8 de junio. Por tanto, la revisión intermedia del MFP está pendiente de su adopción formal en tanto Reino Unido no levante su reserva.

\section{Perspectivas para el marco financiero post-2020}

La negociación del próximo MFP 20212027 se iniciará con la presentación de una propuesta por parte de la Comisión, que de conformidad con el Reglamento 1311/2013 debe hacerse antes de enero de 2018 y sobre su base se desarrollará un proceso de negociación que deberá finalizar con un acuerdo unánime antes de finales de 2020. «En caso contrario, si no se ha adoptado ningún reglamento del Consejo por el que se establezca un nuevo MFP antes del 31 de diciembre de 2020 , los límites máximos y las demás disposiciones correspondientes al último año del marco financiero plurianual deberán prorrogarse hasta que se haya adoptado un reglamento que establezca un nuevo marco financiero plurianual» (artículo 25 del Reglamento 1311/2013).

\subsection{Punto de partida de la Comisión}

La Comisión ha adelantado, en diferentes foros, una serie de principios que guiarán su propuesta de MFP 2021-2027, entre los que destacan el enfoque de un presupuesto comunitario basado en el rendimiento y cumplimiento de resultados, buscando un gasto efectivo y útil en políticas con claro valor añadido UE y potenciando políticas como defensa y seguridad, y la consolidación de una mayor transparencia y simplificación del gasto, sobre todo en la compleja gestión de fondos europeos por parte de los EE MM.

Además, teniendo en cuenta los resultados de la revisión intermedia del MFP 2014-2020, probablemente, dará un paso más en el incremento de la flexibilidad presupuestaria y, a la vez, fomentará las sinergias entre diferentes formas de financiación de políticas (ie: combinación del FEIE con los fondos estructurales), dando preferencia al uso de instrumentos financieros frente al uso de las tradicionales subvenciones (grants, en inglés).

Junto a lo anterior, existen otras cuestiones que se pondrán de manifiesto en las negociaciones a petición expresa del Parlamento Europeo, como, por ejemplo, la duración del MFP, en la actualidad siete años; el TFUE prevé una duración mínima de cinco años, periodo solicitado por el Parlamento en previas negociaciones, a efectos de hacer coincidir el MFP con la duración del mandato parlamentario. Por otro lado, que $\triangleright$ 
se tengan en cuenta las recomendaciones del Grupo de Alto Nivel sobre recursos propios (este último punto se desarrolla posteriormente).

No debe perderse de vista el hecho de que el proceso de negociación de MFP post-2020 se presenta en un escenario muy diferente al vigente durante la negociación del MFP 2014-2020.

Por un lado, se parte de la base de una UE con 27 EE MM y que la negociación del MFP se verá afectada por las negociaciones en paralelo del brexit, que, una vez activado el 29 de marzo por parte de Reino Unido (artículo 50 del Tratado de Lisboa), tendrá una duración de dos años.

Por otro lado, la negociación tendrá en cuenta nuevos factores y circunstancias que no se dieron en la negociación del MFP 20142020 , tales como el hecho de que el nuevo MFP 2021-2027 se enmarca, frente a la situación de crisis del período anterior, en un ambiente de recuperación económica y social en mayor o menor desarrollo en todos los países de la UE. Algunos de ellos continúan con ajustes de gasto y políticas restrictivas, pero, por otro lado, llevando a cabo profundas reformas estructurales que requieren financiación adicional.

\subsection{Sistema de financiación-reforma de los recursos propios}

El MFP presenta una estructura de gasto presupuestario que requiere de un adecuado sistema de financiación a través, principalmente, de los recursos propios.

En 2013, el Consejo acordó la creación del Grupo de Alto Nivel sobre recursos propios, como requisito exigido por el Parlamento Europeo para dar su consentimiento al MFP 2014-2020, y cuyo objeto era realizar un estudio y análisis en profundidad del sistema de financiación de la UE y emitir un informe con las oportunas conclusiones y recomendaciones.

El informe Monti ${ }^{7}$, que fue presentado en el Consejo ECOFIN del 27 de enero de 2017, analiza las deficiencias del sistema de financiación actual, concluyendo la necesidad de llevar a cabo una reforma del mismo y proponiendo una serie de recomendaciones para su mejora. Si bien el informe no tiene carácter vinculante, la Comisión ha reconocido en numerosas ocasiones la importancia del trabajo realizado por el Grupo y ha manifestado su intención de tener en cuenta el contenido del informe a la hora de elaborar su propuesta para el próximo MFP y revisar el sistema de recursos propios.

La reforma, en términos del informe, debería guiarse por los principios de valor añadido europeo, subsidiariedad, neutralidad presupuestaria, no incremento de la carga fiscal, transparencia y, sobre todo, resalta el establecimiento de vínculos entre los ingresos y determinadas políticas de gasto (ie: un impuesto sobre el $\mathrm{CO}_{2}$ que financie políticas medioambientales).

Con un sistema actual de financiación en el que los ingresos de los recursos propios tienen un carácter residual y se basa fundamentalmente en las contribuciones que conforme a su RNB realizan los EE MM al presupuesto UE, el informe recomienda la creación de nuevos recursos propios que incrementen los ingresos y sugiere, entre otros:

- Los relacionados con el mercado único y la coordinación fiscal: un recurso propio procedente del IVA reformado (que sustituya al actual), un impuesto europeo sobre sociedades, un impuesto sobre las transacciones financieras e impuestos sobre otras actividades financieras.

\footnotetext{
7 En referencia al presidente del grupo M. Monti.
} 
- Los relacionados con las políticas sobre la Unión de la Energía, medio ambiente, clima o transporte, que comprenden un impuesto sobre el $\mathrm{CO}_{2}$; los ingresos procedentes del régimen de comercio de derechos de emisión de la UE, un impuesto sobre la electricidad, un impuesto sobre los carburantes (o impuestos especiales sobre los combustibles fósiles en general).

La Comisión deberá tener en cuenta la viabilidad de las recomendaciones del informe en la elaboración de su propuesta para el MFP 2021-2027.

\section{Conclusiones}

La revisión intermedia del MFP 2014-2020 llevada a cabo ha puesto de manifiesto las dificultades presupuestarias (sobre todo en ejecución del presupuesto anual) que conlleva fijar un MFP a siete años, con límites de gasto muy ajustados y una preasignación de créditos con escaso margen de maniobra. Teniendo en cuenta este aspecto, y partiendo de las premisas expuestas con anterioridad, se puede concluir que la negociación del MFP 2021-2027 será compleja. Los 27 EE MM defenderán sus posiciones, las negociaciones se dilatarán en el tiempo, con alta probabilidad, por un periodo de casi dos años, pero los 27 EE MM son conscientes de que deberán alcanzar un acuerdo unánime antes del inicio de 2021. Cabe pensar que mostrarán un espíritu constructivo y favorable a un acuerdo que, por primera vez en la historia de la UE, contará con un Estado miembro menos.

\section{Bibliografía}

[1] COMISIÓN EUROPEA (2007). Tratado de Lisboa por el que se modifican el Tratado de la
Unión Europea y el Tratado Constitutivo de la Comunidad Europea. 2007/C 306/01. Bruselas: Diario Oficial de la Unión Europea. Disponible en: http://eur-lex.europa.eu/legal-content/ES/ TXT/?uri=OJ:C:2007:306:TOC

[2] COMISIÓN EUROPEA (2012). Versión consolidada del Tratado de Funcionamiento de la Unión Europea. 2012 C 326/47. Bruselas: Diario Oficial de la Unión Europea. Disponible en: http://eur-lex.europa.eu/legal-content/ES/TXT/ PDF/?uri=CELEX:12012E/TXT\&from $=E S$

[3] CONSEJO EUROPEO. (2013). Reglamento (UE, EURATOM) n. ${ }^{\circ} 1311 / 2013$ por el que se establece el marco financiero plurianual para el período 2014-2020. Bruselas: Diario Oficial de la Unión Europea. Disponible en: http:// eur-lex.europa.eu/legal-content/ES/TXT/ $P D F /$ ?uri=CELEX:32013R1311\&from $=E S$

[4] PARLAMENTO EUROPEO, CONSEJO Y COMISIÓN EUROPEA (2013). Acuerdo Interinstitucional de 2 de diciembre de 2013, sobre disciplina presupuestaria, cooperación en materia presupuestaria y buena gestión financiera. Bruselas: Diario Oficial de la Unión Europea. Disponible en: http:// eur-lex.europa.eu/LexUriServ/LexUriServ. do?uri=OJ:C:2013:373:0001:0011:ES:PDF

[5] COMISIÓN EUROPEA (2016). Comunicación de la Comisión al Parlamento y al Consejo. Examen/revisión intermedios del marco financiero plurianual 2014-2020. COM(2016) 603 final. Bruselas, 14.09.2016.

[6] COMISIÓN EUROPEA (2016). Future financing of the EU. Final report and recommendations of the High Level Group on Own Resources. Bruselas, diciembre de 2016. Disponible en: $h$ ttp://ec.europa.eu/budget/mff/ hlgor/library/reports-communication/hlgor-report_20170104.pdf

[7] CONSEJO EUROPEO (2017). ST7030/17 y ST7031/17 ADD2. Agreement in principle and statements.

[8] MINISTERIO DE HACIENDA Y FUNCIÓN PÚBLICA (2017). Relaciones financieras entre España y la Unión Europea 2016. Madrid, 09.12.2016.

[9] COMISIÓN EUROPEA (2017). The Budget explained. Bruselas. Disponible en: http:// ec.europa.eu/budget/explained/glossary/ glossary_en.cfm 
Subdirección General de Asuntos Económicos y Financieros

[10] COMISIÓN EUROPEA (2016). Budget focus on results. Bruselas. Disponible en: http://ec.europa.eu/budget/library/budget4results/bfor-conference-2016-p4-overview-panel-4-secondpart-of-mff-2014-2020-and-post-2020.pdf
[11] PARLAMENTO EUROPEO (2017). Briefing EU Legislation in Progress. Bruselas. Disponible en: http://www.europarl.europa.eul RegData/etudes/BRIE/2016/593569/EPRS_ BRI(2016)593569_EN.pdf

ANEXO

MARCO FINANCIERO PLURIANUAL 2014-20120

(Millones de euros corrientes)

\begin{tabular}{|c|c|c|c|c|c|c|c|c|}
\hline Créditos de compromiso & 2014 & 2015 & 2016 & 2017 & 2018 & 2019 & 2020 & Total \\
\hline 1. Crecimiento inteligente e integrador & 52.756 & 77.986 & 69.304 & 73.512 & 76.420 & 79.924 & 83.661 & 513.563 \\
\hline 1.a) Competitividad para el crecimiento y el empleo & 16.560 & 17.666 & 18.467 & 19.925 & 21.239 & 23.082 & 25.191 & 142.130 \\
\hline 1.b) Cohesión económica, social y territorial & 36.196 & 60.320 & 50.837 & 53.587 & 55.181 & 56.842 & 58.470 & 371.433 \\
\hline 2. Crecimiento sostenible: recursos naturales & 49.857 & 64.692 & 64.262 & 60.191 & 60.267 & 60.344 & 60.421 & 420.034 \\
\hline Gastos de mercado y pagos directos & 43.779 & 44.190 & 43.951 & 44.146 & 44.163 & 44.241 & 44.264 & 308.734 \\
\hline 3. Seguridad y ciudadanía & 1.737 & 2.456 & 2.546 & 2.578 & 2.656 & 2.801 & 2.951 & 17.725 \\
\hline 4. Europa global & 8.335 & 8.749 & 9.143 & 9.432 & 9.825 & 10.268 & 10.510 & 66.262 \\
\hline 5. Administración & 8.721 & 9.076 & 9.483 & 9.918 & 10.346 & 10.786 & 11.254 & 69.584 \\
\hline Gasto administrativo de las instituciones & 7.056 & 7.351 & 7.679 & 8.007 & 8.360 & 8.700 & 9.071 & 56.224 \\
\hline 6. Compensaciones & 29 & 0 & 0 & $\mathbf{0}$ & 0 & 0 & 0 & 29 \\
\hline Total créditos de compromiso & 121.435 & 162.959 & 154.738 & 155.631 & 159.514 & 164.123 & 168.797 & 1.087.197 \\
\hline En porcentaje RNB & 0,90 & 1,17 & 1,05 & 1,04 & 1,04 & 1,04 & 1,03 & 1,04 \\
\hline Total créditos de pago & 135.762 & 140.719 & 144.685 & 142.906 & 149.713 & 154.286 & 157.358 & 1.025 .429 \\
\hline En porcentaje RNB & 1,01 & 1,02 & 0,98 & 0,95 & 0,97 & 0,97 & 0,96 & 0,98 \\
\hline Margen disponible (\%) & 0,22 & 0,21 & 0,25 & 0,28 & 0,26 & 0,26 & 0,27 & 0,25 \\
\hline Límite máximo RR PP en porcentaje RNB & 1,23 & 1,23 & 1,23 & 1,23 & 1,23 & 1,23 & 1,23 & 1,23 \\
\hline
\end{tabular}

